\title{
Exceptional points of infinite order give a continuous spectrum
}

\author{
Andrei Smilga \\ SUBATECH, Université de Nantes, \\ 4 rue Alfred Kastler, BP 20722, Nantes 4430\%, France; \\ smilga@subatech.in2p3.fr
}

\begin{abstract}
The statement in the title discussed earlier in association with the Pais-Uhlenbeck oscillator with equal frequencies is illustrated for an elementary matrix model. In the limit $N \rightarrow \infty$ ( $N$ being the order of the exceptional point), an infinity of nontrivial states that do not change their norm during evolution appear. These states have real energies lying in a continuous interval. The norm of the "precursors" of these states at large finite $N$ is not conserved, but the characteristic time scale where this nonconservation shows up grows linearly with $N$.
\end{abstract}


Exceptional points are points in the space of the parameters of the Hamiltonian where two or more eigenstates coalesce such that the Hamiltonian involves a Jordan block at this point [1]. Such a Hamiltonian is not Hermitian, and the corresponding evolution operator is not unitary. Our main remark is that for an exceptional point of infinite order, the Hermiticity and unitarity may in some cases be restored. The spectrum of the Hamiltonian thus obtained is real and continuous.

This phenomenon was earlier observed and studied for the Pais-Uhlenbeck oscillator [2] with the Lagrangian

$$
L_{P U}=\frac{1}{2}\left(\ddot{q}+\Omega_{1}^{2} q\right)\left(\ddot{q}+\Omega_{2}^{2} q\right)
$$

One can observe that the point $\Omega_{1}=\Omega_{2}$ is an exceptional point of infinite order [3] (an infinity of eigenstates that were distinct at $\Omega_{1} \neq \Omega_{2}$ coalesce at this point forming Jordan blocks of infinite size). However, it was later shown [4] that these infinite Jordan blocks do not lead in this case to the loss of Hermiticity and unitarity, but signal the appearance of a continuous spectrum. 1

To avoid possible confusion, we emphasize that the statement above refers to the conventional PU oscillator. Bender and Mannheim recently suggested an unconventional realization of this system [6, 7]. In constrast to the standard PU oscillator Hamiltonian that involves ghosts (the states with arbitrarily low energies), their PT-symmetric Hamiltonian is positive definite. When $\Omega_{1} \neq \Omega_{2}$, both the standard PU Hamiltonian and the BM Hamiltonian are Hermitian. In the singular equal-frequency limit, the dynamics of these two different quantum problems is essentially different. In this limit the BM Hamiltonian does not involve infinite Jordan blocks, but an infinite set of Jordan blocks, each of finite size. The latter break Hermiticity and unitarity [4] (we do not agree with Ref. [7] on this issue). On the other hand, for the conventional PU Hamiltonian, the presence of an infinite number of coalescing states does not lead to the breaking of unitarity.

The aim of this note is to clarify this phenomenon by studying a simple matrix model. Consider the equation

$$
i \frac{d \psi}{d t}=H \psi
$$

where $\psi=\left(\begin{array}{c}a \\ b\end{array}\right)$ is a two-component vector and $H$ has the Jordan form

$$
H=\left(\begin{array}{ll}
0 & 1 \\
0 & 0
\end{array}\right)
$$

A general solution to the equation (2) is

$$
\psi(t)=\left(\begin{array}{c}
a-i b t \\
b
\end{array}\right) .
$$

\footnotetext{
1 The fact that the Hamiltonian of the PU oscillator with equal frequencies has a real continuous spectrum (which, however, is not bounded from below, nor from above) was actually known since the original Pais and Uhlenbeck paper. We can also refer the reader to more recent Ref. [5] for a nice detailed analysis of this issue.
} 
If $b \neq 0$, the (conventionally defined) norm of the state (4) grows with time such that the evolution is not unitary. For the subspace $\psi=\left(\begin{array}{c}a \\ 0\end{array}\right)$, the evolution is unitary, however.

Consider now an exceptional point of order $N$. It can be described by the matrix Schrödinger equation (2) where $\psi(t)$ is now an $N$-component vector and the Hamiltonian represents the matrix [8] 2

$$
H_{N}=\left(\begin{array}{cccccc}
0 & 1 & 0 & 0 & \cdots & 0 \\
0 & 0 & 1 & 0 & \cdots & 0 \\
0 & 0 & 0 & 1 & \cdots & 0 \\
\cdots & \cdots & \cdots & \cdots & \cdots & 1 \\
0 & \cdots & \cdots & \cdots & \cdots & 0
\end{array}\right) .
$$

A general solution to Eq. (2) with the Hamiltonian (5) with the initial conditions

$$
\psi(0)=\left(\begin{array}{c}
a_{0} \\
a_{1} \\
\cdots \\
a_{N-1}
\end{array}\right)
$$

is

$$
\psi(t)=\left(\begin{array}{c}
a_{0}-i a_{1} t-\frac{t^{2}}{2} a_{2}+\cdots+\frac{(-i t)^{N-1}}{(N-1) !} a_{N-1} \\
a_{1}-i a_{2} t-\frac{t^{2}}{2} a_{3}+\cdots+\frac{(-i t)^{N-2}}{(N-2) !} a_{N-1} \\
\cdots \\
a_{N-2}-i a_{N-1} t \\
a_{N-1}
\end{array}\right)
$$

The evolution is unitary only if $a_{1}=\ldots=a_{N-1}=0$. But, generically, it is not.

Let us, however, impose the special initial conditions,

$$
a_{j}=\epsilon^{j}
$$

with a real $\epsilon$. One can observe that in this case the components of the vector (7) represent truncated exponentials,

$$
\psi(t)=\left(\begin{array}{c}
E_{N}(-i \epsilon t) \\
\epsilon E_{N-1}(-i \epsilon t) \\
\cdots \\
\epsilon^{N-1}
\end{array}\right)
$$

where $E_{j}(x)=\sum_{k=0}^{j-1} \frac{x^{k}}{k !}$. In the limit $N \rightarrow \infty$, the exponentials are no longer truncated and the solution (9) goes over to

$$
\psi(t)=\left(\begin{array}{c}
1 \\
\epsilon \\
\cdots
\end{array}\right) e^{-i t \epsilon}
$$

\footnotetext{
${ }^{2}$ A more complicated matrix model for multiple exceptional points was earlier suggested in Ref. [9].
} 
If $|\epsilon|<1$, the norm of this state,

$$
\|\psi(t)\|^{2}=\sum_{j=0}^{\infty} \epsilon^{2 j}=\frac{1}{1-\epsilon^{2}},
$$

is finite and does not depend on time. We can multiply the wave function (10) by $\sqrt{1-\epsilon^{2}}$ to normalize it. Obviously, the parameter $\epsilon$ (it can either be positive or negative) has the meaning of energy.

We have derived that, whereas, at any finite $N$, the evolution is unitary only in a limited 1-dimensional subspace with only one nonvanishing component of the vector $\psi$, for $N \rightarrow \infty$, an infinity of certain special state vectors appear whose norm does not grow with time. It is instructive to explore how this limit is achieved. The norm of the finite $N$ states (9) is

$$
\|\psi(t)\|^{2}=\sum_{j=1}^{N} \epsilon^{2(N-j)}\left|E_{j}(-i \epsilon t)\right|^{2}
$$

If $t$ is not too large, the R.H.S. of Eq. (11) is roughly constant. An elementary qualitative analysis shows that the norm becomes a nontrivial "live" function of time at the characteristic scale

$$
t^{*} \sim \frac{N}{C \epsilon}
$$

with $C \sim 1$. Numerical estimates show that $C \approx 2.5$. We present in Fig. 1 the time dependence of $\|\psi(t)\|$ for $N=10$ and $N=20$ with energy $\epsilon=0.2$.

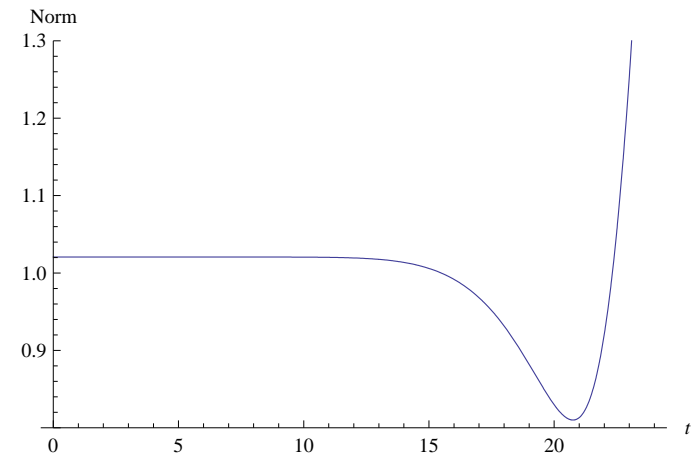

(a) $\mathrm{N}=10$

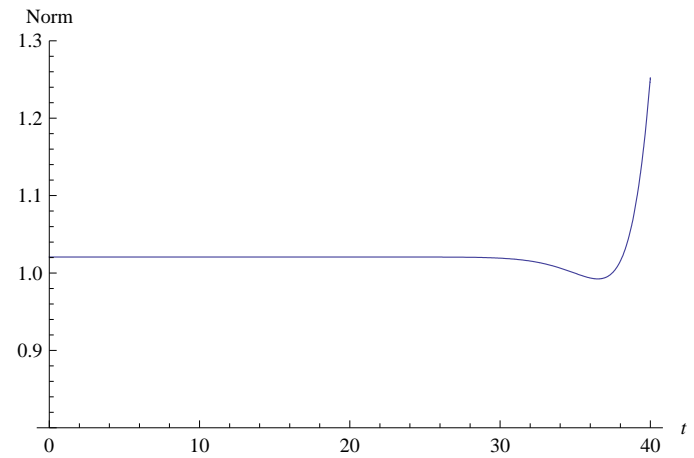

(b) $\mathrm{N}=20$

Figure 1: Norm $\|\psi\|$ of the states (9) with $\epsilon=0.2$ as a function of time.

As far as the $N \rightarrow \infty$ limit of the described matrix model is concerned, one can make the following observations:

1. The states (8) represent a complete basis in the space of normalized vectors (6). Indeed, consider a superposition of the states (8) with the weight function $f(\epsilon)$. The conditions

$$
\int_{-1}^{1} f(\epsilon) \epsilon^{j} d \epsilon=a_{j}
$$

can be resolved by orthogonalizing (13) and representing $f(\epsilon)$ as a series over Legendre polynomials $P_{n}(\epsilon)$. 
2. The existence of the stationary-norm solutions does not mean here that the evolution is unitary for all initial conditions. Indeed, if we start at $t=0$ with the vector $a_{j}=\epsilon^{j}+\mu^{j}$, $\epsilon \neq \mu$, its norm will depend on time. This is a corollary of the fact that the different states (8) are not mutually orthogonal.

3. One can also construct a state whose norm grows exponentially with time. Indeed, impose the initial conditions

$$
a_{j}=\alpha^{j}
$$

with complex $\alpha,|\alpha|<1, \operatorname{Im}(\alpha)>0$. This is an eigenfunction of the Hamiltonian $H^{*}=\lim _{N \rightarrow \infty} H_{N}$ with $H_{N}$ defined in (5) 3

Its time evolution boils down to multiplying by the exponentially growing factor $e^{-i \alpha t}$. One can interpret $\alpha$ as "complex energy". Note that the state (14) can, as any other state, be represented as a superposition of the basis states (8) . The corresponding weight function is

$$
f(\epsilon)=\frac{1}{2} \sum_{n=0}^{\infty}(2 n+1) P_{n}(\epsilon) P_{n}(\alpha) .
$$

4. Nonconservation of the norm for generic initial conditions means that the evolution operator is not unitary and the Hamiltonian is not Hermitian.

5. One can still ask whether the Hamiltonian $H^{*}$ might belong to the class of pseudoHermitian or, better to say, crypto-Hermitian Hamiltonians [10]. Crypto-Hermitian Hamiltonians are Hamiltonians that are not Hermitian, but have a real spectrum. By changing the definition of the norm, they can be rendered manifestly Hermitian.

In our case, the Hamiltonian has eigenvectors with real eigenvalues which constitute a complete basis in the Hilbert space. One might hope to unravel the crypto-Hermitian structure of $H^{*}$ by constructing an approximation to it by finite matrices (cf. [11]) which are different from the original matrix Hamiltonians (5) (the latter are evidently not Hermitian, nor crypto-Hermitian) and are crypto-Hermitian at any finite $N$. For any finite $N$, one could then perform a similarity transformation associated with the norm redefinition making the states orthogonal and the Hamiltonian Hermitian. We attempted to pursue this program in the Appendix. We found out that it does not work - as $N$ grows, the system of $N$ eigenvectors thus constructed becomes less and less independent, the determinant of their components vanishing exponentially fast.

\footnotetext{
${ }^{3}$ In this limit, the Hamiltonian (5) coincides after a similarity transformation with the annihilation operator of a harmonic oscillator,

$$
a=\left(\begin{array}{cccccc}
0 & 1 & 0 & 0 & \ldots & 0 \\
0 & 0 & \sqrt{2} & 0 & \ldots & 0 \\
0 & 0 & 0 & \sqrt{3} & \ldots & 0 \\
\ldots & \ldots & \ldots & \ldots & \ldots & \ldots
\end{array}\right)
$$

The eigenstates (14) are then nothing but coherent states. To avoid confusion, bear in mind, however, that, unlike the annihilation operator (15), the Hamiltonian (5) is responsible, as any Hamiltonian is, for the time evolution of the system.
} 
Thus, even though one can construct finite-N crypto-Hermitian matrix approximants, the limit $N \rightarrow \infty$ is singular.

On the other hand, for the PU oscillator with equal frequencies, we have an ordinary continuous spectrum with a unitary evolution operator. An essential difference of this system from the matrix model studied in this paper is the fact that the Jordan block structure appears there in the basis of "bad" unnormalizable states. (When $\Omega_{1} \neq \Omega_{2}$, the corresponding states are quite "good", belonging to $\mathcal{L}_{2}$. But in the equal-frequency limit, the exponential factor rendering them normalizable disappears.)

We refer the reader to Refs. [3, 4] for a more detailed analysis of this system and only discuss here, following [4, a trivial model with the same physics. Consider the Hamiltonian describing free 1-dimensional motion,

$$
H=-\frac{1}{2} \frac{\partial^{2}}{\partial x^{2}}
$$

It has the continuous spectrum eigenfunctions

$$
\Psi_{k}(x ; t)=\exp \left\{i k x-\frac{i k^{2}}{2} t\right\}
$$

Note now that not only (18), but also every term of its expansion in $k$,

$$
\begin{aligned}
& k^{0}: \Psi_{0}(x ; t)=1, \\
& k^{1}: \Psi_{1}(x ; t)=x, \\
& k^{2}: \Psi_{2}(x ; t)=t-i x^{2}, \\
& k^{3}: \Psi_{3}(x ; t)=x t-\frac{i x^{3}}{3}, \\
& k^{4}: \Psi_{4}(x ; t)=\frac{t^{2}}{2}-i t x^{2}-\frac{x^{4}}{6},
\end{aligned}
$$

etc., satisfies the time-dependent Schrödinger equation (2) with the Hamiltonian (17). Obviously, the functions (19) are not normalizable. Still, one can observe that the Hamiltonian (17) expressed in this unusual basis acquires a nondiagonal Jordan-like form, 4

$$
H \Psi_{0}=H \Psi_{1}=0, \quad H \Psi_{2}=i \Psi_{0}, \quad H \Psi_{3}=i \Psi_{1}, \quad H \Psi_{4}=i \Psi_{2}, \text { etc. }
$$

and one can relate the continuity of the spectrum of (17) to this fact. "Bad" (not normalizable) basis leads to "good" [mutually orthogonal in the usual sense, $\int \Psi_{k^{\prime}}^{*} \Psi_{k} d x \sim \delta\left(k^{\prime}-k\right)$ ] continuous spectrum eigenfunctions.

And, for the matrix model, "good" (normalizable) basis states (8) result in "bad" continuous spectrum states - nonorthogonal real-energy states and also states with complex energies.

I am indebted to W.D. Heiss for useful discussions and to U. Guenther, H.F. Jones and M. Znojil for many illuminating discussions and valuable comments.

\footnotetext{
${ }^{4}$ The Hilbert space of the Hamiltonian (17) has two sectors with the functions even and odd under $x \rightarrow-x$. Correspondingly, we have here two Jordan ladders.
} 


\section{Appendix}

We explain here why an attempt to construct a sequence of crypto-Hermitian matrix Hamiltonians with the same limit $N \rightarrow \infty$ as the limit $H^{*}$ of the Hamiltonians (5) fails.

Consider the normalized eigenstates of $H^{*},|\epsilon\rangle: a_{j}=\sqrt{1-\epsilon^{2}} \epsilon^{j}$ with real $\epsilon \in(-1,1)$. We have

$$
\langle\epsilon \mid \mu\rangle=\frac{\sqrt{\left(1-\epsilon^{2}\right)\left(1-\mu^{2}\right)}}{1-\epsilon \mu} .
$$

Consider now a set of $N=2 M+1 N$-dimensional unitary vectors $|n=-M, \ldots, 0, \ldots, M\rangle$ with the inner products

$$
\langle n \mid m\rangle=\frac{\sqrt{\left(1-\epsilon_{n}^{2}\right)\left(1-\epsilon_{m}^{2}\right)}}{1-\epsilon_{n} \epsilon_{m}}, \quad \epsilon_{n}=\frac{n}{M+1} .
$$

For example, for $M=1$, we choose

$$
|-\rangle=\left(\begin{array}{c}
\frac{\sqrt{3}}{2} \\
\frac{1}{\sqrt{20}} \\
-\frac{1}{\sqrt{5}}
\end{array}\right), \quad|0\rangle=\left(\begin{array}{l}
1 \\
0 \\
0
\end{array}\right), \quad|+\rangle=\left(\begin{array}{c}
\frac{\sqrt{3}}{2} \\
\frac{1}{\sqrt{20}} \\
\frac{1}{\sqrt{5}}
\end{array}\right) .
$$

Obviously, (A.2) represents a discretization of (A.1).

At the next step, we construct the matrix $(\tilde{H})_{N}$ having the vectors $|n\rangle$ as eigenvectors with eigenvalues $\epsilon_{n}$. This is possible to do as long as the system of $N^{2}$ equations

$$
\left(\tilde{H}_{N}\right)_{i j} a_{j}^{(n)}=\epsilon_{n} a_{i}^{(n)}
$$

for the matrix elements $\left(\tilde{H}_{N}\right)_{i j}$ is not degenerate. In other words, as long as the vectors $|n\rangle$ are linearly independent and the determinant of the $N \times N$ matrix made of the vector components does not vanish. For $M=1$, this determinant is equal to 0.2. This indicates that, though the vectors (A.3) are not coplanar, they are relatively close to being so. Finally, one can make these vectors orthogonal by redefining the norm in an appropriate way.

However, the larger $M$ and $N$ are, the more difficult is to carry on this program. It is more convenient to study not the determinant of the eigenvector components, but its square, the Gram determinant of the matrix of their scalar products. For $M=1$, the Gram deteminant $\Delta_{1}$ of the scalar products (A.2) is equal to 0.04 . For $M=2$, it is equal to $10^{-5}$, meaning that the corresponding five eigenvectors are "almost" linearly dependent. For $M=3$, it is already $10^{-11}$. It roughly decays as $\Delta_{M} \sim e^{-1.2 M^{2}}$. 5 A matrix with an almost degenerate system of eigenvectors must have very large elements. The limit $N \rightarrow \infty$ is singular.

\section{References}

[1] For a review, see [W.D. Heiss, J. Phys. A 37 (2004) 2455, quant-ph/0304152].

[2] A. Pais and G.E. Uhlenbeck, Phys. Rev. 79 (1950) 145.

\footnotetext{
${ }^{5}$ To rigorously justify this numerical observation is an interesting problem for a mathematics student.
} 
[3] A.V. Smilga, Phys. Lett. B 632 (2006) 433, hep-th/0503213.

[4] A.V. Smilga, SIGMA 5 (2009) 017 , arXiv:0808.0139 [quant-ph].

[5] K. Bolonek and P. Kosinski, quant-ph/0612009.

[6] C. Bender and P. Mannheim, Phys. Rev. Lett. 100 (2008) 110402, arXiv:0706.0207 [hepth].

[7] C. Bender and P. Mannheim, Phys. Rev. D 78 (2008) 025022, arXiv:0804.4190 [hep-th].

[8] W.D. Heiss, talk at the Int. Conf. PHHQP-14, Setif (Algeria), Sept. 5-10, 2014.

[9] M. Znojil, J. Phys. A 40 (2007) 4863, math-ph/0703070.

[10] See e.g. F.G. Scholtz, H.B. Geyer, and F.J.W. Hahne, Ann. of Phys. (NY) 213 (1992) 74; C.M. Bender and S. Boettcher, Phys. Rev. Lett. 80 (1998) 5243, physics/9712001; A. Mostafazadeh, J. Math. Phys. 43 (2002) 2814, math-ph/0110006.

[11] G. Levai, F. Ruzicka and M.Znojil, Int J. Theor. Phys. 53 (2014) 2875, arXiv: 1403.0723 [quant-ph]. 